\title{
BMJ Open OPTIMAL and ENSURE trials-based combined cost-effectiveness analysis of erlotinib versus chemotherapy for the first-line treatment of Asian patients with non-squamous non-small-cell lung cancer
}

Feng Wen, ${ }^{1,2}$ Hanrui Zheng, ${ }^{2,3}$ Pengfei Zhang, ${ }^{2,3}$ David Hutton, ${ }^{4}$ Qiu Li ${ }^{1,2}$

To cite: Wen $\mathrm{F}$, Zheng $\mathrm{H}$, Zhang P, et al. OPTIMAL and ENSURE trials-based combined cost-effectiveness analysis of erlotinib versus chemotherapy for the first-line treatment of Asian patients with non-squamous non-smallcell lung cancer. BMJ Open 2018;8:e020128. doi:10.1136/ bmjopen-2017-020128

- Prepublication history and additional material for this paper are available online. To view these files, please visit the journal online (http://dx.doi org/10.1136/bmjopen-2017020128).

FW and $\mathrm{HZ}$ contributed equally.

Received 19 0ctober 2017 Revised 4 February 2018 Accepted 22 February 2018

Check for updates

${ }^{1}$ Department of Medical Oncology, Cancer Center, West China Hospital, Sichuan University, Chengdu, China ${ }^{2}$ West China Biostatistics and Cost-Benefit Analysis Center, Sichuan University, Chengdu, China

${ }^{3}$ Department of Clinical Pharmacy, West China Hospital, Sichuan University, Chengdu,

China

${ }^{4}$ Department of Health

Management and Policy,

University of Michigan, Ann

Arbor, Michigan, USA

Correspondence to

Professor Qiu Li;

keythera@126.com

\section{ABSTRACT}

Objectives Erlotinib, the first generation of epidermoid growth factor receptor-tyrosine kinase inhibitor (EGFR-TKI), has been recommended as an essential treatment in patients with nonsmall-cell lung cancer (NSCLC) with EGFR mutation. Although it has improved progression-free survival (PFS), overall survival (OS) was limited and erlotinib can be expensive. This costeffectiveness analysis compares erlotinib monotherapy with gemcitabine-included doublet chemotherapy.

Setting First-line treatment of Asian patients with NSCLC with EGFR mutation.

Methods A Markov model was created based on the results of the ENSURE (NCT01342965) and OPTIMAL (CTONG-0802) trials which evaluated erlotinib and chemotherapy. The model simulates cancer progression and all causes of death. All medical costs were calculated from the perspective of the Chinese healthcare system.

Main outcome measures The primary outcomes are costs, quality-adjusted life years (QALYs) and incremental cost-effectiveness ratios (ICERs).

Results The combined PFS was 11.81 months and 5.1 months for erlotinib and chemotherapy, respectively, while the OS was reversed at 24.68 months for erlotinib and 26.16 months for chemotherapy. The chemotherapy arm gained 0.13 QALYs compared with erlotinib monotherapy (1.17 QALYs vs 1.04 QALYs), while erlotinib had lower costs ( $\$ 55230$ vs $\$ 77669$ ), resulting in an ICER of $\$ 174808$ per QALY for the chemotherapy arm, which exceeds three times the Chinese GDP per capita. The most influential factors were the health utility of PFS, the cost of erlotinib and the health utility of progressed disease.

Conclusion Erlotinib monotherapy may be acceptable as a cost-effective first-line treatment for NSCLC compared with gemcitabine-based chemotherapy. The results were robust to changes in assumptions.

Trial registration number NCT01342965 and CTONG-0802.

\section{INTRODUCTION}

Lung cancer, the most prevalent cancer worldwide, ranks first in cancer-related death

\section{Strengths and limitations of this study}

- This is the first study to address both the overall health and economic impact of erlotinib monotherapy compared with gemcitabine-included doublet chemotherapy as first-line treatment of Asian patients with NSCLC with epidermoid growth factor receptor mutation based on two independent phase III, randomised, open-label trials, but not on patient-level data.

- Utilities used in the current model are based on previous published research, but uncertainty in these utilities may influence the calculation of effectiveness.

- The detailed information of treatments after progression and adverse events induced in the subsequent therapy was limited.

among men and is the second leading cause of cancer death among women, causing $27 \%$ of all cancer deaths. ${ }^{1}$ Notably, about $50 \%$ of lung cancer deaths each year happen in low-income and middle-income countries. The estimated rates of new cancer cases and deaths for China were 733 and 610 per 100000 in 2015, respectively. ${ }^{2}$ However, the prognosis of lung cancer is frustrating. Most patients with non-small-cell lung cancer (NSCLC) suffer from progression within 3-6 months with the treatment of standard chemotherapy, and the overall survival (OS) is limited to 10 months. ${ }^{3}$ Even with new agents added to the therapeutic armamentarium, the 5-year survival of less than $20 \%$ is still disappointingly low, meaning lung cancer is still a major public health problem. ${ }^{4}$

Currently, the ongoing development of epidermoid growth factor receptor-tyrosine kinase inhibitor (EGFR-TKI) has spurred 
more treatment options for patients with NSCLC with EGFR mutation-positive disease in addition to the traditional platinum-based doublet chemotherapy, for example, either carboplatin or cisplatin combined with a third-generation cytotoxic agent (paclitaxel, gemcitabine, pemetrexed, docetaxel and vinorelbine)..$^{5}$ As the first generation of orally administered EGFR-TKI agents, erlotinib (Tarceva) has created dramatic responses in sensitising patients with EGFR mutation NSCLC since the American FDA approved it as the second-line regimen in 2005.

Of note, EFGR mutation prevalence is higher in Asian populations, with a rate of up to $30 \%$ compared with $8 \%-16 \%$ in Caucasian patients. ${ }^{6-8}$ However, both populations benefit from the EGFR-TKI treatments. A European phase III study, EURTAC, suggested erlotinib achieved a significant progression-free survival (PFS) improvement for the first-line treatment of patients with EGFR mutation-positive NSCLC compared with chemotherapy (9.7 months vs 5.2 months). ${ }^{9}$ Meanwhile, another two independent phase III, randomised, open-label studies have been completed in Asian populations, the ENSURE trial (NCT01342965) and the OPTIMAL trial (CTONG-0802). Both studies demonstrated that erlotinib provided significant improvement in PFS compared with chemotherapy (13.1 months vs 4.6 months in the OPTIMAL trial; 11.0 months vs 5.5 months in the ENSURE trial)..$^{5} 1011$ Based on the above results, Asian patients may benefit more with erlotinib treatment than Caucasian patients.

However, the OS benefit of first-line erlotinib was not as significant as PFS. Additionally, the survival outcomes were inconsistent even in the two Asian clinical trials. It was reported that erlotinib gained 0.8 months more than chemotherapy in the ENSURE Study (26.3 months vs 25.5 months), but the OS was 4.4 months shorter for erlotinib compared with chemotherapy in the OPTIMAL Study (22.8 months vs 27.2 months). This puts decision makers in a dilemma when choosing an optimal strategy for patients with NSCLC. ${ }^{511}$

In addition to understanding the trade-offs between PFS and OS, clinicians and policy makers are also interested in financial considerations of this important therapy choice as both erlotinib and chemotherapy are expensive. This cost-effectiveness analysis aims to provide new insights into the health and financial implications of erlotinib monotherapy compared with gemcitabine-included doublet chemotherapy in the first-line treatment of Asian patients with EGFR mutation NSCLC as viewed from the perspective of the Chinese healthcare system.

\section{MATERIALS AND METHODS}

This cost-effectiveness analysis uses a mathematical model approach to evaluate the overall costs and health outcomes of patients under each therapy option. The Markov model tracks costs and health outcomes by simulating progression and death based on the results of the trials. Costs and health utilities are associated with the therapies as well as progression-free and progressed disease. The Markov model approach allows for simulation of results beyond the lengths observed in the trial. In addition, the model can allow for sensitivity analysis to examine how results might be different with different parameter values.

\section{Overall concept of the Markov model}

TreeAge software (TreeAge, Williamstown, Massachusetts, USA) was used to build a Markov decision model evaluating the economic consequences and therapeutic efficacy associated with the treatment strategies. The model simulated monthly transition cycles over 10 years, which incorporates the entire life span of most patients. Figure 1 presents the model states and transitions. The costs were calculated from a Chinese health system perspective, and health outcomes were reported in quality-adjusted life years (QALYs). Incremental cost-effectiveness ratios (ICERs), defined as cost per unit of health outcome gained, were calculated as the difference in incremental costs divided by the difference in incremental health effectiveness between a given strategy and the next most costly alternative. As the survivals in both groups were short, discount rates were not considered in the study.

\section{The strategies and the Markov model structure}

Two groups were analysed that mimic the treatment strategies in the two Asian studies: in group 1, patients with advanced NSCLC were treated with erlotinib monotherapy; in group 2, patients with advanced NSCLC were treated with gemcitabine-included doublet chemotherapy. The Markov model included three clinical states: PFS, progressive disease (PD) and death (figure 1). Patients were assumed to enter the model in the PFS state, and eased because of progression of disease, intolerable toxicities or death. Importantly, after progression of disease, second-line treatment was switched (and predefined in the ENSURE trial) to chemotherapy for the erlotinib arm and erlotinib for the chemotherapy arm.

\section{Progression data from the trials: patient characteristics}

Two independent phase III, randomised, open-label trials enrolled 382 Asian patients, 193 in the erlotinib group and 189 in the chemotherapy group. Twenty-two centres in China were included in the OPTIMAL Study from 24 August 2008 to 7 July 2009, and 30 centres across China, Malaysia and the Philippines were analysed in the ENSURE trial from March 2011 to June 2012. All the eligible participants were patients with stage IIIB or stage IV advanced NSCLC with Eastern Cooperative Oncology Group Performance Status (ECOG PS) of $0-2$. All the patients were older than 18 years old with an EGFR mutation (exon 19 deletion or exon 21 L858R point mutation). The detailed information of the two studies is listed in table 1 . 


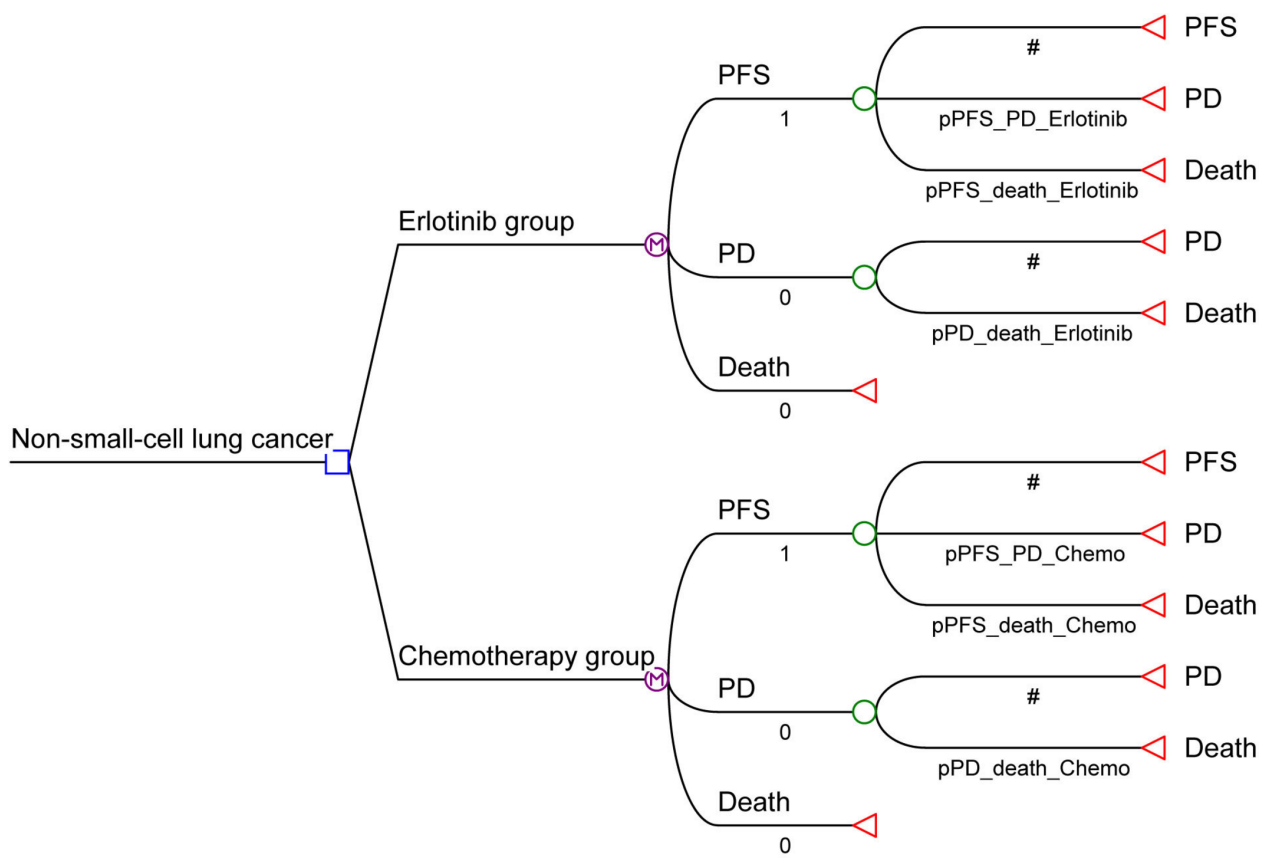

Figure 1 Markov model for cost-effectiveness analysis of erlotinib versus chemotherapy for the first-line treatment of Asian patients with non-squamous NSCLC. Two groups were analysed: group 1, patients with advanced NSCLC treated with erlotinib monotherapy; group 2, patients with advanced NSCLC treated with gemcitabine-included doublet chemotherapy. A Markov model comprising three health states (PFS, PD and death) was built. Chemo, chemotherapy; NSCLC, non-small cell lung cancer; PD, progressive disease; PFS, progression-free survival; pPD_death_Chemo, probability of PD state to death for chemotherapy arm; pPD_death_Erlotinib, probability of PD state to death for Erlotinib arm; pPFS_death_Chemo, probability of PFS state to death for chemotherapy arm; pPFS_death_Erlotinib, probability of PFS state to death for Erlotinib arm; pPFS_PD_ Chemo, probability of PFS state to PD for chemotherapy arm; pPFS_PD_Erlotinib, probability of PFS state to PD for Erlotinib arm.

\section{The treatments}

Patients in the erlotinib group received oral erlotinib $(150 \mathrm{mg} /$ day) until disease progression or unacceptable toxicity. Patients in the chemotherapy group received up to four cycles of gemcitabine plus carboplatin (gemcitabine $1000 \mathrm{mg} / \mathrm{m}^{2}$ intravenous on days 1 and 8 , carboplatin (area under the curve $=5$ ) intravenous on day 1 of a 3-week cycle) and cisplatin (gemcitabine $1250 \mathrm{mg} / \mathrm{m}^{2}$ intravenous on days 1 and 8 , cisplatin $75 \mathrm{mg} / \mathrm{m}^{2}$ intravenous on day 1 of a 3-week cycle) in the OPTIMAL trial and ENSURE trial, respectively. After disease progression, therapies were switched.

\section{Clinical outcomes}

Efficacy was evaluated every 6 weeks including regular physical examination, blood tests, tumour marker exams and CT, MRI and bone scans for the target tumour lesions. Tumour efficacy evaluations were classified according to RECIST V.1.0 and V.1.1 for the OPTIMAL Study and ENSURE Study, respectively. Adverse events (AEs) were assessed based on National Cancer Institute Common Terminology Criteria for AEs V.3.0 and V.4.0, respectively.

Both studies demonstrated erlotinib provided significant improvement in PFS compared with chemotherapy (13.1 months vs 4.6 months in the OPTIMAL trial; 11.0 months vs 5.5 months in the ENSURE trial). In terms of OS, the differences of both studies were not statistically significant. Erlotinib gained 0.8 months more than chemotherapy in the ENSURE Study (26.3 months vs 25.5 months), but the OS was 4.4 months shorter with erlotinib compared with chemotherapy in the OPTIMAL Study (22.8 months vs 27.2 months).

The state translation probabilities were combined from both studies weighted by the sample size of each study. The combined PFS was 11.81 months and 5.1 months for erlotinib and chemotherapy, respectively, while the OS was much closer, 24.68 months for erlotinib and 26.16 months for chemotherapy. The transition probabilities of health states were estimated based on the equation: $p(1$ month $)=1-(0.5)^{(1 /}$ median time to event) 1213 and calibration. The computed monthly transition probabilities from PFS to PD (pPFS-PD), from PFS to dead (pPFS-dead) and from PD to dead (pPD-dead) are described in online supplementary appendix table 1 and the related distributions used in sensitivity analysis are listed in online supplementary appendix table 2.

\section{Health utilities}

Time spent in each health state was weighted by a utility score to calculate the total QALYs associated with each treatment. Preference-based utility scores were derived from previously published literature and the values were set at $0.65(0.26-$ $0.87)$ for PFS state with erlotinib and $0.56(0.42-0.7)$ for PFS with chemotherapy because of the toxicity. ${ }^{14-16}$ The utility of PD was $0.47(0.19-0.58)$ and 0 for dead for both groups. ${ }^{14-16}$ The related distributions are listed in online supplementary appendix table 2. 
Table 1 Information from the OPTIMAL and ENSURE trials

\section{OPTIMAL \\ (CTONG-0802, NCT00874419)}

ENSURE

\begin{tabular}{|c|c|c|c|c|}
\hline & & & \multirow{2}{*}{\multicolumn{2}{|c|}{ Ann Oncol 2015}} \\
\hline Publication(s) & \multicolumn{2}{|c|}{ Lancet Oncol 2011; Ann Oncol 2015} & & \\
\hline Design & \multicolumn{2}{|c|}{$\begin{array}{l}\text { Multicentre, phase III, randomly assigned } \\
(1: 1) \\
\text { First-line treatment }\end{array}$} & \multicolumn{2}{|c|}{$\begin{array}{l}\text { Multicentre, phase III, randomly assigned (1:1) } \\
\text { First-line treatment }\end{array}$} \\
\hline Experimental & \multicolumn{2}{|c|}{ Erlotinib $150 \mathrm{mg} /$ day } & \multicolumn{2}{|c|}{ Erlotinib 150 mg/day } \\
\hline Active comparator & \multicolumn{2}{|c|}{ Gemcitabine+carboplatin up to four cycles } & \multicolumn{2}{|c|}{ Gemcitabine+cisplatin up to four cycles } \\
\hline Primary outcome & \multicolumn{2}{|c|}{ PFS } & \multicolumn{2}{|c|}{ PFS } \\
\hline $\begin{array}{l}\text { Response evaluation } \\
\text { criteria }\end{array}$ & \multicolumn{2}{|l|}{ RECIST V.1.0 } & \multicolumn{2}{|c|}{ RECIST V.1.1 } \\
\hline Criteria for $\mathrm{AE}$ record & \multicolumn{2}{|l|}{ NCI-CTCAE V.3.0 } & \multicolumn{2}{|c|}{ NCI-CTCAE V.4.0 } \\
\hline Population & \multicolumn{2}{|c|}{$\begin{array}{l}22 \text { centres in China, } \\
\text { advanced NSCLC (stage IIIB or stage IV) } \\
\text { ECOG PS: } 0-2\end{array}$} & \multicolumn{2}{|c|}{$\begin{array}{l}30 \text { centres across China, Malaysia and the Philippines, } \\
\text { advanced NSCLC (stage IIIB or stage IV) } \\
\text { ECOG PS: } 0-2\end{array}$} \\
\hline Age & \multicolumn{2}{|c|}{ Older than 18years } & \multicolumn{2}{|c|}{ Older than 18 years } \\
\hline EGFR mutation & \multicolumn{2}{|c|}{$\begin{array}{l}\text { Exon } 19 \text { deletion or exon } 21 \text { L858R point } \\
\text { mutation }\end{array}$} & \multicolumn{2}{|c|}{$\begin{array}{l}\text { Exon } 19 \text { deletion or exon } 21 \\
\text { L858R mutation }\end{array}$} \\
\hline \multirow[t]{2}{*}{ Time period } & \multicolumn{2}{|c|}{24 August 2008-17 July 2009} & \multicolumn{2}{|c|}{ March 2011-June 2012} \\
\hline & Erlotinib & Chemotherapy & Erlotinib & Chemotherapy \\
\hline N & 83 & 82 & 110 & 107 \\
\hline Evaluable Pts & 82 & 72 & 110 & 107 (safety 104) \\
\hline mPFS (m), 95\% Cl & $\begin{array}{l}13.1 \\
(10 \cdot 58 \text { to } 16 \cdot 53)\end{array}$ & $\begin{array}{l}4.6 \\
(4 \cdot 21 \text { to } 5 \cdot 42)\end{array}$ & 11.0 & 5.5 \\
\hline $\mathrm{HR}, 95 \% \mathrm{Cl}$ & \multicolumn{2}{|l|}{$0.16(0.10$ to 0.26$)$} & \multicolumn{2}{|c|}{0.34 (0.22 to 0.51$)$} \\
\hline$P$ value & \multicolumn{2}{|l|}{$<0.0001$} & \multicolumn{2}{|l|}{$<0.0001$} \\
\hline $\mathrm{mOS}(\mathrm{m}) 95 \% \mathrm{Cl}$ & 22.8 & 27.2 & 26.3 & 25.5 \\
\hline $\mathrm{HR}, 95 \% \mathrm{Cl}$ & \multicolumn{2}{|l|}{1.19 (0.83 to 1.71$)$} & \multicolumn{2}{|c|}{0.91 (0.63 to 1.31$)$} \\
\hline$P$ value & \multicolumn{2}{|l|}{0.2663} & \multicolumn{2}{|c|}{0.607} \\
\hline AEs & \multicolumn{2}{|l|}{ Favours erlotinib } & \multicolumn{2}{|c|}{ Favours erlotinib } \\
\hline
\end{tabular}

(NCT01342965)

AEs, adverse events; ECOG PS, Eastern Cooperative Oncology Group Performance Status; EGFR, epidermoid growth factor receptor; m, median; N, number; NCl-CTCAE, National Cancer Institute Common Terminology Criteria for Adverse Events; NSCLC, non-small cell lung cancer; OS, overall survival; PFS, progression-free survival; Pts, patients.

\section{Costs}

Costs in the PFS and PD states included costs of therapies (erlotinib/chemotherapy and supportive drugs), costs of grade 3-4 AEs, costs of hospitalisation, and costs of tests for efficacy and toxicities/safety during the treatments. Treatment-associated costs are listed in online supplementary appendix table 1 and distributions are listed in online supplementary appendix table 2. Costs for the first-line therapies as well as second-line treatments were included in the analysis. Detailed data on the grade 3-4 AEs were derived from the records of the original studies. ${ }^{5}{ }^{1011}$ Costs were calculated from a Chinese health system perspective and all costs were converted into US dollars, with an exchange rate of $\$ 1=¥ 6.58$ (21 February 2016).

\section{Sensitivity analysis}

One-way sensitivity analysis was performed to examine the impact of uncertainty in parameter values on the results.
The factors were varied according to 95\% CIs found in the literature (utility of PFS and PD state), or if such CIs were not available, they were increased or decreased by $20 \%{ }^{17}$ According to the WHO guidelines for cost-effective analysis, the willingness-to-pay (WTP) threshold for cost-effectiveness was set to $\$ 24048$ per year, which was $3 \times$ Gross domestic product (GDP) per capita of China in 2015. ${ }^{18} 19$ In addition, probabilistic sensitivity analysis was performed by conducting a second-order Monte Carlo simulation to estimate the optimal probability of each strategy under varying WTP thresholds (see online supplementary appendix table 2). ${ }^{17}$

\section{RESULTS}

Effectiveness

Initiating therapy with chemotherapy had 0.13 more QALYs compared with initiating with erlotinib 
Table 2 Results of the cost-effectiveness analysis

\begin{tabular}{lll}
\hline & Erlotinib & Chemotherapy \\
\hline Costs(\$) & & \\
\hline PFS state(\$) & 34258.48 & 7938.54 \\
\hline PD state(\$) & 20971.85 & 69730.00 \\
\hline Total (\$) & 55230.34 & 77668.54 \\
\hline $\begin{array}{l}\text { Incremental costs(\$) } \\
\text { Effectiveness(QALYs) }\end{array}$ & - & 22438.20 \\
\hline PFS state & 0.59 & 0.28 \\
\hline PD state & 0.44 & 0.89 \\
\hline Total & 1.04 & 1.17 \\
\hline $\begin{array}{l}\text { Incremental effectiveness } \\
\text { Incremental cost/ } \\
\text { effectiveness(\$) }\end{array}$ & & 0.13 \\
\hline
\end{tabular}

PD, progressive disease; PFS, progression-free survival; QALY, quality-adjusted life month.

monotherapy first (1.17 QALYs vs 1.04 QALYs). Erlotinib-first had 0.59 QALYs accrued in the PFS state and 0.44 QALYs accrued in the PD state. Chemotherapy-first had 0.28 QALYs in the PFS state and 0.89 QALYs in the PD state. The details are listed in table 2.

\section{Costs}

Costs for the erlotinib agent were much more expensive than gemcitabine-included doublet chemotherapy (\$2766 vs \$519). Consequently, costs while in the PFS state were higher with erlotinib than with chemotherapy (\$34258 vs $\$ 7939)$. However, costs for the PD state were higher in the chemotherapy arm than in the erlotinib arm (\$69730 vs \$20972), because more patients in chemotherapy received subsequent therapy, of whom most patients chose EGFR-TKI or other chemotherapies. The constitutions of subsequent therapy of both groups were listed in online supplementary appendix table 3 summarised from the primary studies. Costs of
AEs were cheaper in the erlotinib arm compared with chemotherapy arm ( $\$ 0.20$ vs $\$ 28.78$, table 3$)$. In total, the chemotherapy-first arm had an incremental cost of $\$ 22438$ over the erlotinib-first arm (\$55230 vs $\$ 77,669)$.

\section{Cost-effectiveness analysis}

Erlotinib had lower costs, but lower QALYs than chemotherapy. The 0.13 QALYs gained for chemotherapy cost an incremental of $\$ 174808$ per QALY versus erlotinib (figure 2), which exceeds the WTP of \$24048 per QALY for China.

\section{Sensitivity analysis}

One-way sensitivity analysis was conducted to test the responsiveness of the model and the robustness of our results and is shown in the tornado diagram in figure 3. Changes of individual parameters did slightly alter the overall value associated with therapy, but they did not alter the conclusions about the relative cost-effectiveness of chemotherapy versus erlotinib. Changes in the health utility with PFS with erlotinib (uPFS_Erlotinib) had the greatest impact on the results followed by the price of erlotinib (cErlotinib_1), the health utility with PD ( $\mathrm{UPD}$ ), the second-line treatment cost in the erlotinib group (cErlotinib_2) and the costs of tests with erlotinib (cTest_erlotinib). One-way sensitivity was conducted to further determine the effect of these parameters. When the value of the utility for the PFS state with erlotinib changed from 0.26 to 0.78 , the ICER for chemotherapy compared with erlotinib ranged from $\$ 46316$ per QALY to $\$ 4110591$ per QALY; when the value of the utility for the PFS state with erlotinib rose above 0.78 , chemotherapy was dominated by erlotinib. When the cost of erlotinib increased from $\$ 2212$ to $\$ 3318$, the ICER for chemotherapy decreased from $\$ 222023$ per QALY to $\$ 127593$ per QALY. For all of the parameters varied, the ICER for chemotherapy always exceeded the WTP of $\$ 24048$ per QALY for China.

Table 3 Average adverse costs of patients with advanced non-small-cell lung cancer treated with erlotinib or chemotherapy per patient per cycle

\begin{tabular}{llllc}
\hline & Erlotinib (193) & Chemotherapy (176) & Cost of erlotinib(\$) & Cost of chemotherapy(\$) \\
\hline Neutropenia & 1 & 32 & 0.05 & 3.76 \\
Leucopenia & 1 & 22 & 0.05 & 2.58 \\
Anaemia & 1 & 22 & 0.02 & 1.01 \\
Thrombocytopenia & 0 & 36 & 0.00 & 21.39 \\
Vomiting or nausea & 0 & 1 & 0.00 & 0.00 \\
Increased ALT & 3 & 1 & 0.05 & 0.04 \\
Diarrhoea & 1 & 0 & 0.00 & 0.00 \\
Infection & 1 & 0 & 0.02 & 0.00 \\
Stomatitis & 1 & 0 & 0.02 & 0.00 \\
Total & - & - & 0.20 & 28.78 \\
\hline
\end{tabular}




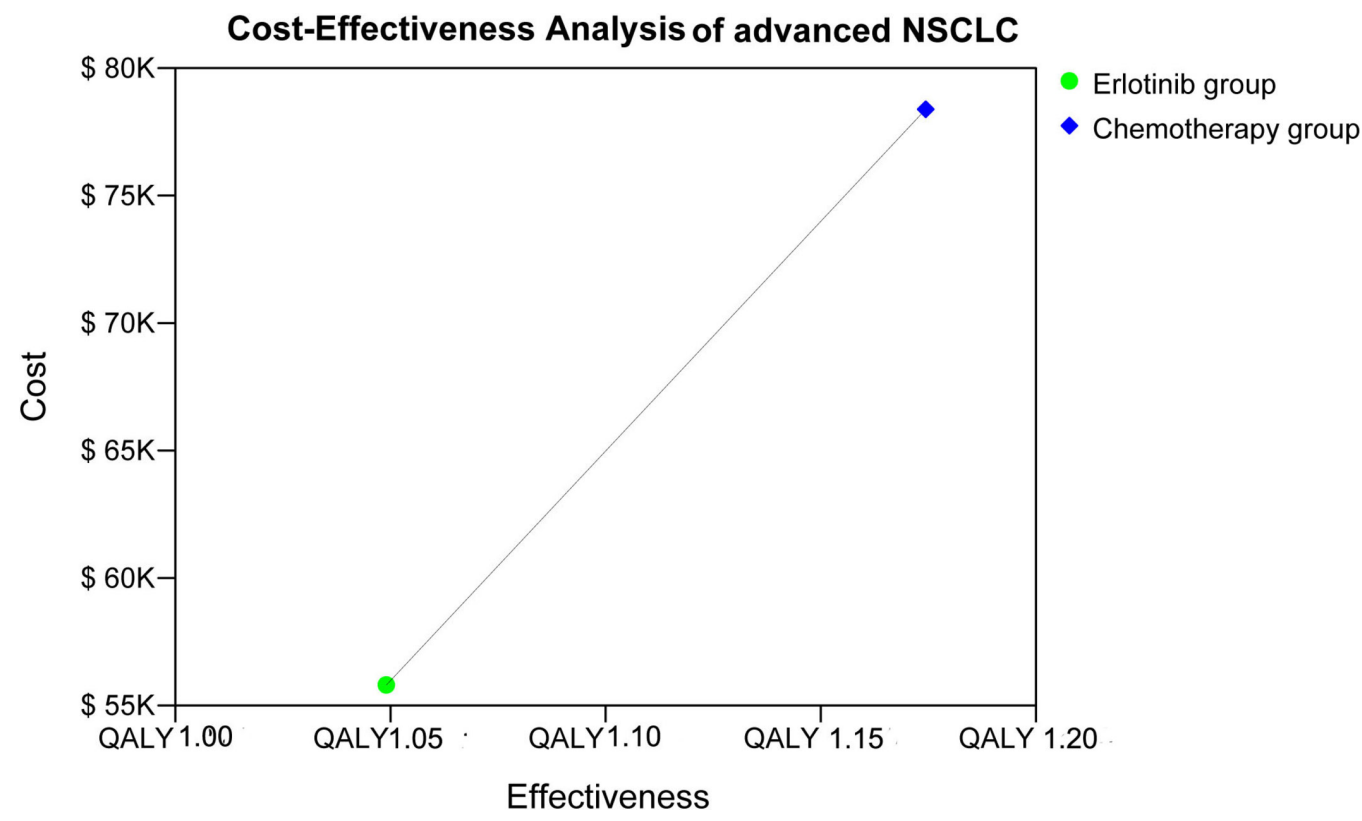

Figure 2 Cost-effectiveness pictured with two groups. Two groups were analysed: group 1, patients with advanced NSCLC treated with erlotinib monotherapy; group 2, patients with advanced NSCLC treated with gemcitabine-included doublet chemotherapy. NSCLC, non-small-cell lung cancer; QALY, quality-adjusted life year.

Additionally, to determine how cost-effectiveness was affected by each clinical trial, scenario analyses were performed where the results of the OPTIMAL and ENSURE trials were used separately (online supplementary figure 1). Specifically, if only the results from the OPTIMAL trial were used to inform the effects of therapy, chemotherapy gained 0.29 QALYs compared with erlotinib with \$33261 higher costs,

Tornado Diagram at Non-small-cell lung cancer

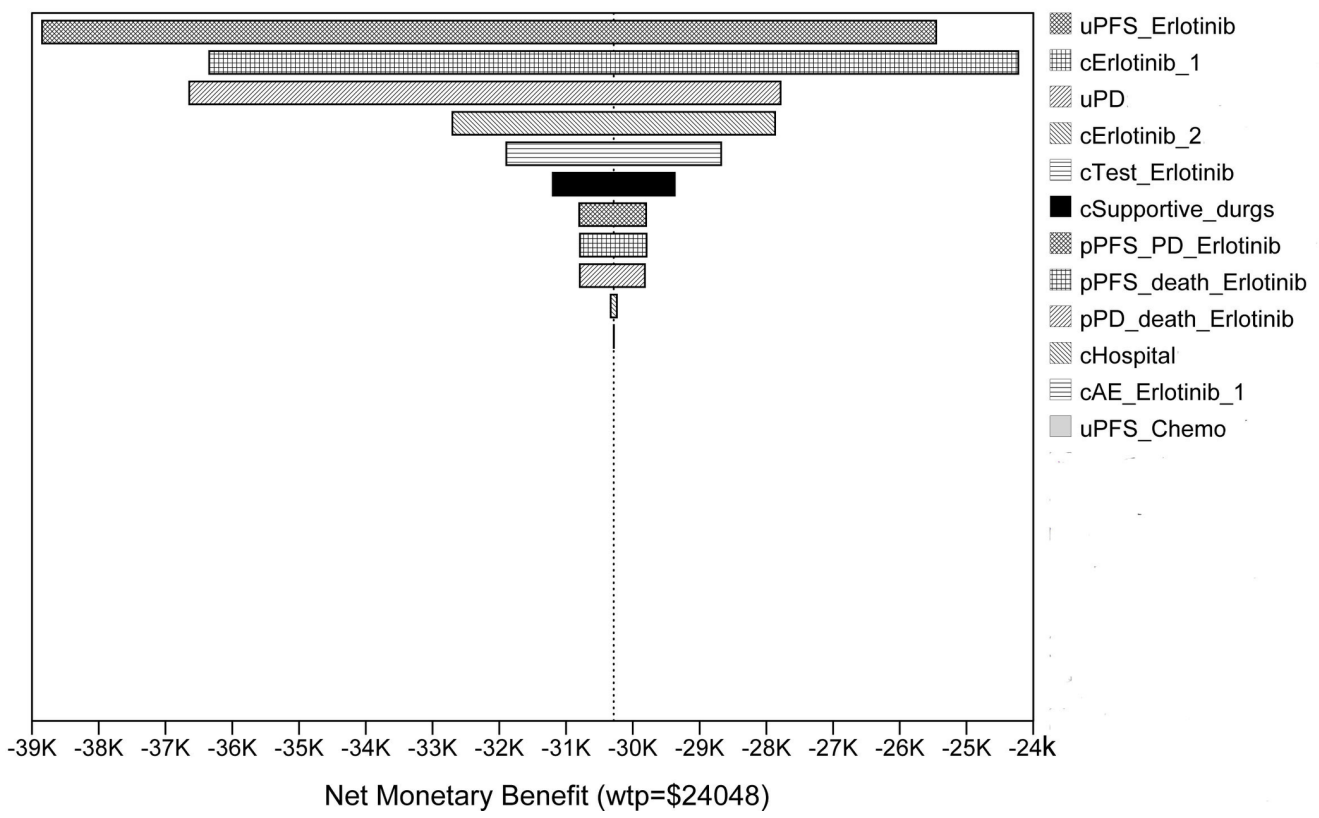

Figure 3 Tornado diagram of one-way sensitivity analysis. A tornado diagram summarises the results of one-way sensitivity analysis to identify model variables associated with the value of strategies in the treatment of advanced non-small cell lung cancer. The most influential factors are at the top of the diagram: going from the most influential to the least: cAE_Erlotinib_1, first-line adverse event costs for Erlotinib arm; cErlotinib_1, first-line erlotinib costs; cErlotinib_2, second-line erlotinib costs; cHospital, hospital expenses; cSupportive_drug, supportive drug costs; cTest_Erlotinib, test costs for erlotinib arm; PD, progressive disease; PFS, progression-free survival; pPD_death_Erlotinib, probability of PD state to death for erlotinib arm; pPFS_death_Erlotinib, probability of PFS state to death for erlotinib arm; pPFS_PD_Erlotinib, probability of PFS state to PD state for erlotinib arm; uPD, health utility of PD state; uPFS_Chemo, health utility of PFS state for chemotherapy arm; uPFS_ Erlotinib, health utility of PFS state for erlotinib arm; WTP, willingness-to-pay. 


\section{Acceptability Curve}

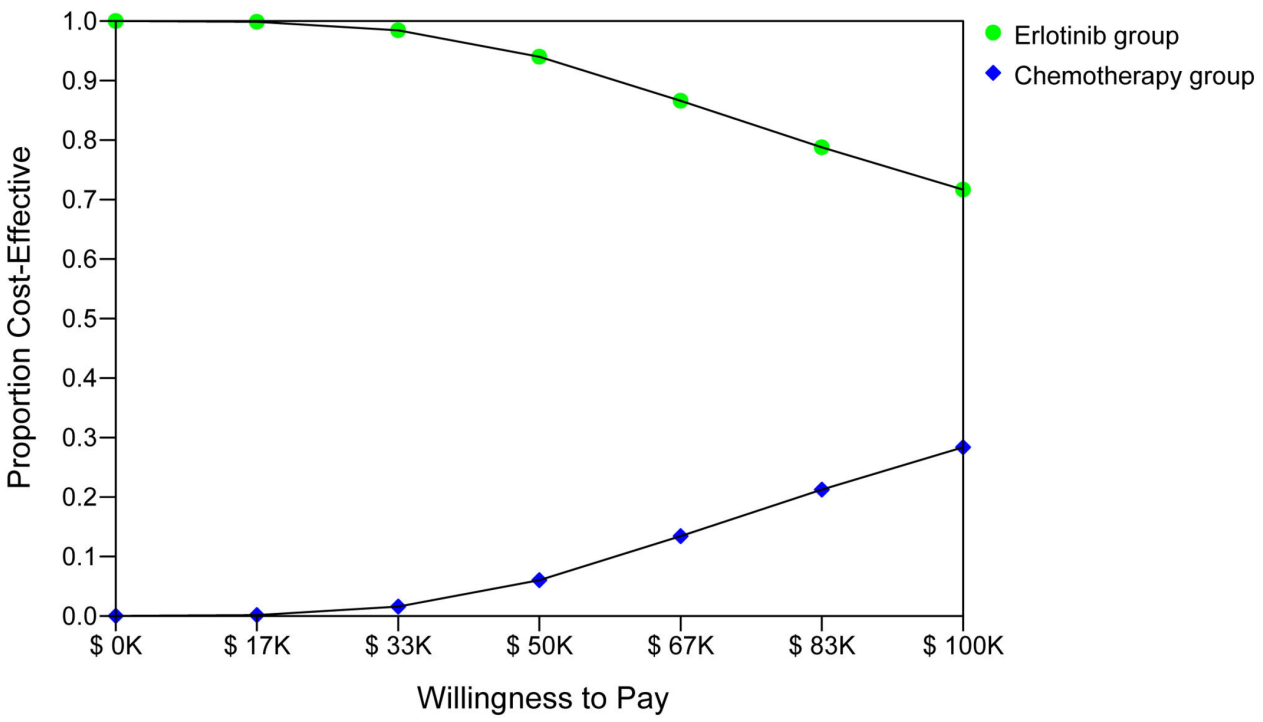

Figure 4 Probabilistic sensitivity analysis (acceptability frontier). The cost-effectiveness acceptability frontier shows the probability of each strategy being cost-effective for different willingness-to-pay thresholds for quality-adjusted life years gained.

leading to an ICER for chemotherapy of \$115 123.5 per QALY gained compared with erlotinib. When using parameters only from the ENSURE trial, chemotherapy was dominated by erlotinib because chemotherapy had higher costs with fewer QALYs. Both scenario analyses suggested that erlotinib would be a cost-effective choice for advanced EGFR-mutated NSCLC.

\section{Probabilistic sensitivity analysis}

The cost-effectiveness acceptability curve from the probabilistic sensitivity analysis shows the probability of strategies being cost-effective for different WTP thresholds. Erlotinib was more likely to be cost-effective compared with chemotherapy, but as the WTP value increased to $\$ 100000$ per QALY, erlotinib was $75 \%$ likely to be cost-effective, while chemotherapy was $25 \%$ likely to be cost-effective (figure 4).

\section{DISCUSSION}

NSCLC incurs a high burden, particularly in developing countries. Although significant progression has been made with the appearance of new cytotoxic drugs and targeted agents, the prognosis of NSCLC is still disappointing and none of these treatment options appear clearly preferred over others. ${ }^{20-22}$ To determine the optimal therapies clinicians and policy makers must consider both the overall health and financial outcomes.

Many economic analyses have focused on chemotherapies as the first-line treatment of advanced NSCLC. It was suggested that the average costs of cisplatin and gemcitabine combinations were lower or similar to other cisplatin or carboplatin combinations, but the differences of efficacy were not significant. ${ }^{23}$ Nevertheless, cost-effectiveness comparisons between targeted therapies, such as erlotinib, and standard chemotherapies are limited. The expensive cost of erlotinib may prohibit extensive clinical application, particularly in developing economies. Hence, both physicians and healthcare regulators urgently need new insights into the costs and health benefits of erlotinib.

To our knowledge, this is the first study to address both the overall health and economic impacts of erlotinib monotherapy compared with gemcitabine-included doublet chemotherapy in the first-line treatment of EGFR mutation in Asian patients with NSCLC based on two independent phase III, randomised, openlabel trials, OPTIMAL and ENSURE. The current study showed the combined PFS was 11.81 months and 5.1 months for erlotinib and chemotherapy, respectively, calculated from the original two trials, while the OS were much closer, 24.68 months for erlotinib and 26.16 months for chemotherapy. The chemotherapy arm had more QALYs than erlotinib, but at an incremental cost of $\$ 174808$ per QALY gained, which exceeds most recommended thresholds of cost-effectiveness for China. We found erlotinib monotherapy to be a more cost-effective first-line treatment for NSCLC compared with gemcitabine-based chemotherapy, the results of which were confirmed by another cost-effectiveness analysis based on the OPTIMAL trial only. ${ }^{15}$

Because the erlotinib-based PFS benefits did not translate into significant OS improvement, the QALYs were lower. The poststudy treatments of both trials may be a contributor to the lack of significant OS improvement. Specifically, the proportion receiving subsequent treatment was higher in the groups initially receiving chemotherapy than the groups initially receiving erlotinib (online supplementary appendix table 3). Based on the OPTIMAL trial, the OS was prolonged in 
patients received second-line treatment compared with those who gave up subsequent therapy, which led to the confounded survival effects. ${ }^{10}$ Additionally, the cost of second-line chemotherapy in the erlotinib group was also an important factor in the model, which emphasised the importance of subsequent treatment beyond first-line therapy.

However, the sensitivity analysis showed that none of the parameter value changes made chemotherapy cost-effective under a threshold of $\$ 24048$ per QALY. Both scenario analyses using data from the OPTIMAL and ENSURE trials individually also conclude that erlotinib would be a cost-effective choice for advanced EGFR-mutated NSCLC when compared with chemotherapy. What's more, the OPTIMAL trial provided evidence that EGFR-TKI treated patients benefited from a longer OS than patients who only had chemotherapy. ${ }^{10}$ In addition, erlotinib is an oral small molecular agent, the delivery of which may be more convenient for patients without the worry of hospital-related charges.

This research has several limitations also. First, the analysis was based on the publicly reported information of two Asian studies, but was not a patient-level data. Nevertheless, our study embraces the combined information from a broad Asian population and was able to track aggregate population outcomes of PFS and OS. This study also examined the medical benefits based on the cost-effectiveness analysis of China's healthcare system. Second, utilities for PFS and PD are based on previous published research, but uncertainty in these utilities may influence the calculation of QALYs. The results would have been more precise if EQ-5D data from the patients with NSCLC treated with erlotinib would have been collected directly. Third, the detailed information of second-line treatments and related AEs induced in the subsequent therapy was limited. The data used in the model were deduced from the two published trials. But, more precise conclusions could be made with a detailed poststudy follow-up information on clinical practice.

In all, this is the first cost-effectiveness analysis to compare the cost-effectiveness of erlotinib monotreatment and gemcitabine-included doublet chemotherapy as first-line treatment of Asian patients with EGFR mutation NSCLC based on two combined clinical trials. Our results suggest that providing erlotinib monotherapy first may be more cost-effective. Decision makers should continue to study patients' health status and subsequent treatments to determine which strategy is more cost-effective.

Contributors Conception/design: QL, FW and DH. Provision of study material or patients: QL, FW and HZ. Collection and/or assembly of data: FW and HZ. Data analysis and interpretation: FW, HZ, DH and PZ. Manuscript writing: FW and $\mathrm{HZ}$. Final approval of the manuscript: all authors.

Funding This research received no specific grant from any funding agency in the public, commercial or not-for-profit sectors.

Competing interests None declared.
Patient consent Not required.

Ethics approval All procedures performed in studies involving human participants were in accordance with the ethical standards of medical ethics committee of West China Hospital, Sichuan University. Additionally, the study was performed in accordance with the ethical standards put forth in the 1964 Declaration of Helsinki.

Provenance and peer review Not commissioned; externally peer reviewed.

Data sharing statement All data are available to the public. We are not interested in participating in the study of examining data sharing.

Open Access This is an Open Access article distributed in accordance with the Creative Commons Attribution Non Commercial (CC BY-NC 4.0) license, which permits others to distribute, remix, adapt, build upon this work non-commercially, and license their derivative works on different terms, provided the original work is properly cited and the use is non-commercial. See: http://creativecommons.org/ licenses/by-nc/4.0/

(c) Article author(s) (or their employer(s) unless otherwise stated in the text of the article) 2018. All rights reserved. No commercial use is permitted unless otherwise expressly granted.

\section{REFERENCES}

1. Torre LA, Siegel RL, Jemal A. Lung cancer statistics. Adv Exp Med Biol 2016;893:1-19.

2. Chen W, Zheng R, Baade PD, et al. Cancer statistics in China, 2015. CA Cancer J Clin 2016;66:115-32.

3. Sandler A, Gray R, Perry MC, et al. Paclitaxel-carboplatin alone or with bevacizumab for non-small-cell lung cancer. N Engl J Med 2006;355:2542-50.

4. Siegel RL, Miller KD, Jemal A. Cancer statistics, 2016. CA Cancer J Clin 2016;66:7-30.

5. Zhou C, Wu YL, Chen G, et al. Final overall survival results from a randomised, phase III study of erlotinib versus chemotherapy as firstline treatment of EGFR mutation-positive advanced non-small-cell lung cancer (OPTIMAL, CTONG-0802). Ann Oncol 2015;26:1877-83.

6. Rosell R, Moran T, Queralt C, et al. Screening for epidermal growth factor receptor mutations in lung cancer. $N$ Engl $J$ Med 2009;361:958-67.

7. Shigematsu H, Lin L, Takahashi T, et al. Clinical and biological features associated with epidermal growth factor receptor gene mutations in lung cancers. $J$ Nat/ Cancer Inst 2005;97:339-46.

8. Wu YL, Zhong WZ, Li LY, et al. Epidermal growth factor receptor mutations and their correlation with gefitinib therapy in patients with non-small cell lung cancer: a meta-analysis based on updated individual patient data from six medical centers in mainland China. $J$ Thorac Oncol 2007;2:430-9.

9. Rosell R, Carcereny E, Gervais R, et al. Erlotinib versus standard chemotherapy as first-line treatment for European patients with advanced EGFR mutation-positive non-small-cell lung cancer (EURTAC): a multicentre, open-label, randomised phase 3 trial. Lancet Oncol 2012;13:239-46.

10. Zhou C, Wu YL, Chen G, et al. Erlotinib versus chemotherapy as first-line treatment for patients with advanced EGFR mutationpositive non-small-cell lung cancer (OPTIMAL, CTONG-0802): a multicentre, open-label, randomised, phase 3 study. Lancet Oncol 2011;12:735-42.

11. Wu YL, Zhou C, Liam CK, et al. First-line erlotinib versus gemcitabine/cisplatin in patients with advanced EGFR mutationpositive non-small-cell lung cancer: analyses from the phase III, randomized, open-label, ENSURE study. Ann Oncol 2015;26:1883-9.

12. Wen F, Yang Y, Zhang P, et al. Cost-effectiveness of RAS screening before monoclonal antibodies therapy in metastatic colorectal cancer based on FIRE3 Study. Cancer Biol Ther 2015;16:1577-84.

13. Petrou PK, Talias MA. Cost-effectiveness of sorafenib compared to best supportive care in second line renal cell cancer from a payer perspective in Cyprus. Expert Rev Pharmacoecon Outcomes Res 2014;14:131-8.

14. Nafees B, Stafford M, Gavriel S, et al. Health state utilities for non small cell lung cancer. Health Qual Life Outcomes 2008;6:84.

15. Wang S, Peng L, Li J, et al. A trial-based cost-effectiveness analysis of erlotinib alone versus platinum-based doublet chemotherapy as first-line therapy for Eastern Asian nonsquamous non-small-cell lung cancer. PLoS One 2013;8:e55917. 
16. Carlson JJ, Garrison LP, Ramsey SD, et al. The potential clinical and economic outcomes of pharmacogenomic approaches to EGFRtyrosine kinase inhibitor therapy in non-small-cell lung cancer. Value Health 2009:12:20-7.

17. Elbasha EH, Messonnier ML. Cost-effectiveness analysis and health care resource allocation: decision rules under variable returns to scale. Health Econ 2004;13:21-35.

18. Murray CJ, Evans DB, Acharya A, et al. Development of WHO guidelines on generalized cost-effectiveness analysis. Health Econ 2000;9:235-51.

19. Eichler HG, Kong SX, Gerth WC, et al. Use of cost-effectiveness analysis in health-care resource allocation decision-making: how are cost-effectiveness thresholds expected to emerge? Value Health $2004 ; 7: 518-28$
20. Schiller JH, Harrington D, Belani CP, et al. Comparison of four chemotherapy regimens for advanced non-small-cell lung cancer. $N$ Engl J Med 2002;346:92-8.

21. Scagliotti G, Brodowicz T, Shepherd FA, et al. Treatment-by-histology interaction analyses in three phase III trials show superiority of pemetrexed in nonsquamous non-small cell lung cancer. $J$ Thorac Oncol 2011;6:64-70.

22. Goffin J, Lacchetti C, Ellis PM, et al. First-line systemic chemotherapy in the treatment of advanced non-small cell lung cancer: a systematic review. J Thorac Oncol 2010;5:260-74.

23. Schiller J, Tilden D, Aristides M, et al. Retrospective cost analysis of gemcitabine in combination with cisplatin in non-small cell lung cancer compared to other combination therapies in Europe. Lung Cancer 2004;43:101-12. 